\title{
Music Synchronizer with Runner's Pace for Supporting Steady Pace Jogging
}

\author{
Tetsuro Kitahara, Shunsuke Hokari, and Tatsuya Nagayasu \\ College of Humanities and Sciences, Nihon University \\ 3-25-40, Sakurajosui, Setagaya-ku, Tokyo 156-8550, Japan \\ \{kitahara,hokari,nagayasu\}@kthrlab.jp
}

\begin{abstract}
This paper describes a music player that automatically synchronizes the music playback speed with jogging pace. The importance of jogging is in running long distances at a steady pace, so it will be useful to alert when the pace varies. Focusing on the fact that a number of people jog while listening to music, we propose a method for telling the runner the pace variation by synchronizing the music playback speed with the runner's pace. Experimental results show that the proposed method facilitates to keep the runner's steady pace.
\end{abstract}

\section{Introduction}

Jogging is an effective way to increase physical fitness [1-4]. The importance of jogging is in running long distances at a steady pace, but this may not be easy for novices. Beginner runners sometimes run too fast and/or decrease their pace due to tiredness. If computing technologies can alert these runners of their pace variations to help them keep a steady pace, it would be useful in allowing them to enjoy jogging.

We focus on the fact that a number of people jog while listening to music with their portable music players (including smartphones). We then propose a method for telling the runner the pace variation by synchronizing the music playback speed with the runner's pace. After the runner's standard pace is measured, the playback speed automatically increases or decreases, according to the runner's pace as it increases or decreases. This method requires the runner to keep the standard pace in order to enjoy the music at its normal speed, therefore we expect that this method will spontaneously influence the runner to keep a steady pace.

There have been some attempts to support jogging using music. Rubisch et al.[5] developed a mobile-phone-based system that recommends musical pieces that have a close tempo to the target heart rate defined by the user-desired amount of oxygen consumption. Sakata et al.[6] proposed a concept called interactive jogging, in which the runner's pace is feedbacked as the change of music. These are similar concepts to ours, but their effects have not yet been confirmed through experiments. In this paper, we investigate the effects of our method by conducting experiments.

C. Stephanidis (Ed.): HCII 2014 Posters, Part II, CCIS 435, pp. 343-348, 2014.

(C) Springer International Publishing Switzerland 2014 


\section{Proposed System}

The user wears an Android device on his/her hip using a belt and jogs while listening to music using the earphones or headphones connected to the Android device. After the music starts, it is then played back at the normal speed for 15 seconds, because the former five seconds are regarded as a setup time of running and the latter ten seconds are used for measuring the runner's standard pace. After measuring the standard pace, the system starts to synchronize the playback speed with the user's pace based on the method described below.

\subsection{Preparation of Audio Signals}

Audio signals of the target music with different playback speeds are generated on a PC in advance. In the current implementation, those with $0.5,0.6, \ldots$, and 2.0 times of the original speed are generated based on Phase Vocoder 7. using MARY Text To Speech Libary 8 .

\subsection{Measurement of Jogging Pace}

The pace is measured with the acceleration sensor built in the Android device. The acceleration value $\alpha(t)$ defined by

$$
\alpha(t)=\left|a_{x}(t)-a_{x}(t-1)\right|+\left|a_{y}(t)-a_{y}(t-1)\right|+\left|a_{z}(t)-a_{z}(t-1)\right|
$$

is measured for every $80 \mathrm{~ms}$, where $a_{x}(t), a_{y}(t)$, and $a_{z}(t)$ are the accelerations along the $\mathrm{x}-, \mathrm{y}_{-}$, and $\mathrm{z}$-axes, respectively. When $\alpha(t)$ is higher than an experimentally determined threshold $\alpha_{\theta}$, the step counter is incremented. The number of steps for the last four seconds is regarded as the jogging pace at that time.

\subsection{Switching of Playback Speed}

For every second, the system calculates the pace and accordingly switches the audio signal. As an example, when the pace at time $t$ is 0.8 times the standard pace, the audio signal with 0.8 times of the normal speed is used.

\section{Experiments}

\subsection{Experimental Condition}

We conducted experiments on jogging using our system. There were ten participants, ages 22 to 23. Participant E exercises every month and Participant $\mathrm{H}$ exercises more than three times a week, while the remaining eight partcipants usually do not exercise. The procedure was as follows:

1. Try a two-minute jog three times, listening to music with the normal playback speed, at 30-s intervals.

2. Rest for enough time.

3. Try a two-minute jog three times again, listening to music with our system, at 30 -s intervals.

To avoid order effects, we replaced Step 1 with Step 3 for half of the participants. 

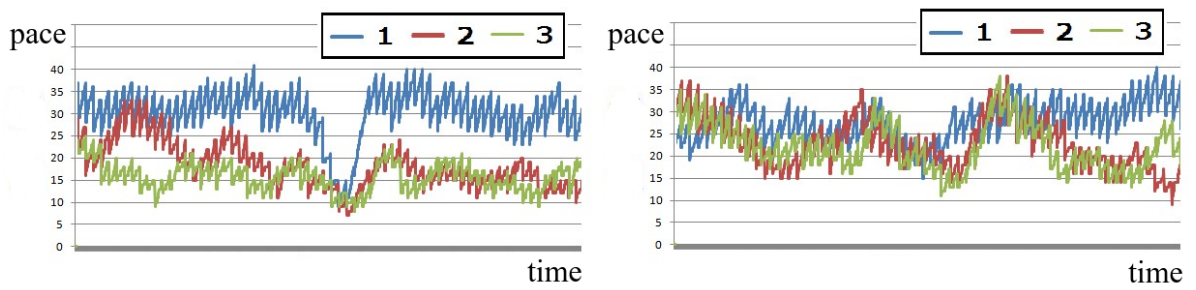

Fig. 1. Pace by Participant A with normal playback (left) and with our system (right)

We used a straight course and asked the participants to turn back around one minute after they started. We used "Tonight's the Night for Love" taken from RWC Music Database [8] and Acer ICONIA TAB A100 with Android 3.2.1.

\subsection{Experimental Results}

Table 1 lists the temporal average and standard deviation of the every-second jogging pace for each trial. Because the data for Participants I and J were lost due to device trouble, those were removed from the table. The results are summarized as follows:

1. Participant A's pace decreased at every trial with normal speed playback. Participant B's pace also varied between the trials. On the other hand, they jogged at consistent paces with our system.

Table 1. Temporal average and standard deviation of pace for each trial

(a) Our System

\begin{tabular}{lllllll}
\hline Participant & \multicolumn{2}{l}{ Trial } & \multicolumn{3}{c}{ Trial 2} & \multicolumn{2}{c}{ Trial 3} \\
\hline A & 28.0 & 2.0 & 21.0 & 2.0 & 21.0 & 3.0 \\
B & 21.5 & 4.0 & 23.8 & 3.9 & 22.4 & 3.0 \\
C & 22.8 & 4.0 & 22.4 & 3.6 & 21.4 & 3.4 \\
D & 27.5 & 3.4 & 30.0 & 3.7 & 29.6 & 3.4 \\
E & 30.2 & 5.1 & 27.7 & 3.9 & 28.8 & 5.0 \\
F & 11.1 & 2.0 & 12.0 & 2.3 & 11.2 & 1.8 \\
G & 27.0 & 4.1 & 22.8 & 3.5 & 19.4 & 3.3 \\
H & 24.7 & 3.8 & 29.5 & 5.1 & 30.1 & 4.6 \\
\hline
\end{tabular}

(b) Normal Speed Playback

\begin{tabular}{lrrrrrr}
\hline Participant & \multicolumn{2}{c}{ Trial 1 } & \multicolumn{3}{c}{ Trial 2 } & \multicolumn{2}{c}{ Trial 3} \\
\hline A & 29.5 & 0.5 & 22.5 & 10.5 & 15.5 & 3.5 \\
B & 10.4 & 2.8 & 20.9 & 5.3 & 24.2 & 3.6 \\
C & 26.4 & 4.3 & 23.2 & 4.3 & 23.3 & 4.2 \\
D & 26.2 & 3.5 & 26.3 & 3.7 & 25.7 & 3.5 \\
E & 27.2 & 5.1 & 26.1 & 4.1 & 27.4 & 4.2 \\
F & 10.8 & 1.6 & 12.5 & 3.7 & 12.7 & 2.3 \\
G & 19.3 & 3.9 & 19.4 & 4.1 & 18.6 & 3.1 \\
H & 8.2 & 2.1 & 21.8 & 4.0 & 24.6 & 4.9 \\
\hline
\end{tabular}




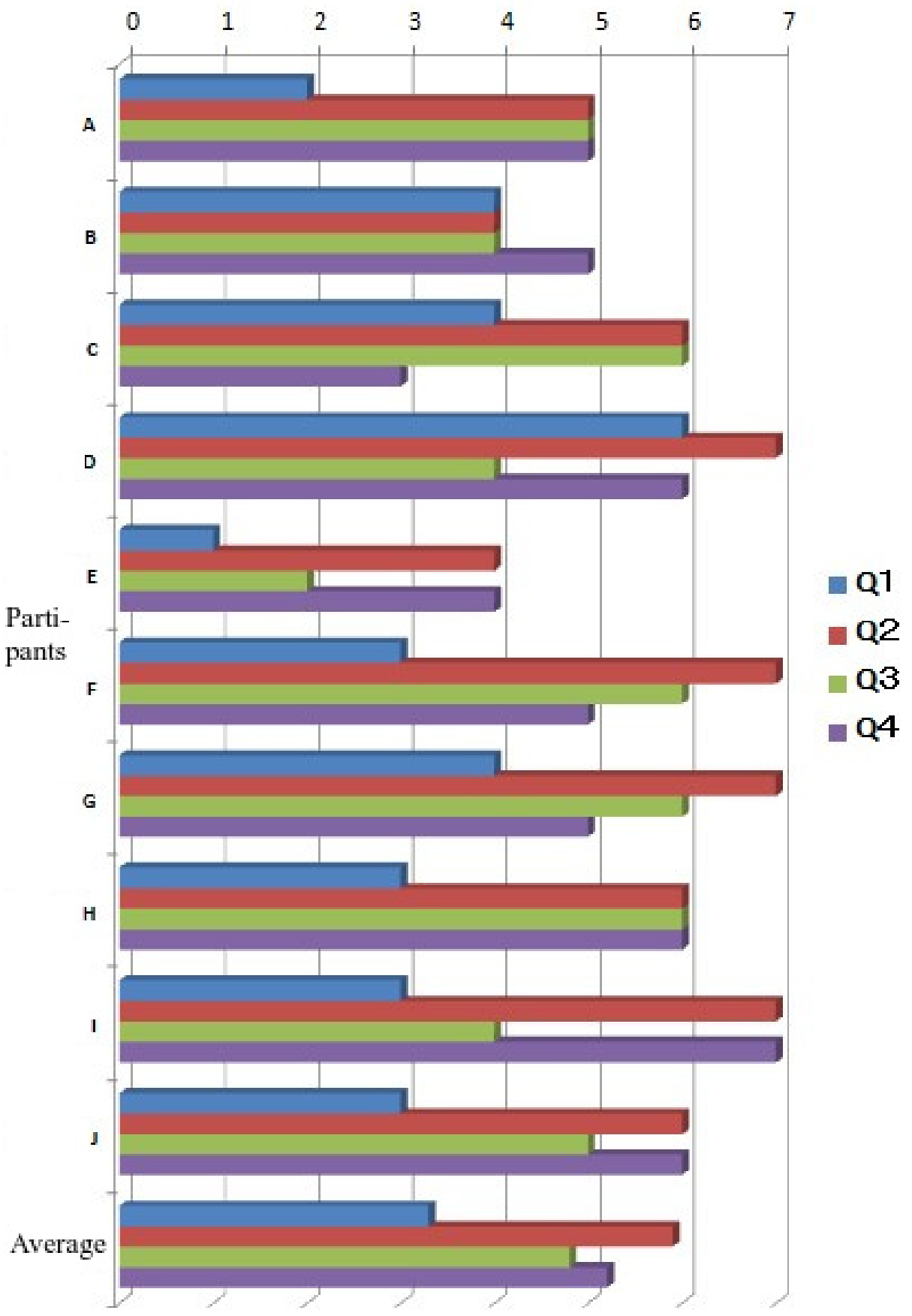

Fig. 2. Results of questionnarie 
2. The standard deviations for the 2nd and 3rd trials were smaller with our system than with normal speed playback except for Participants E and $\mathrm{H}$. This means that our system reduced pace variation caused by tiredness to some extent. The reason why this tendency was not shown in Participans E and $\mathrm{H}$ was that they frequently exercise unlike the other participants.

Details of every-second paces by Participant A are shown in Figure 11, The pace was made lower each trial with normal speed playback, while the pace with our system was not made lower. The pace largely decreased at turning back for normal speed playback, while the pace did not decrease with our system. This would be an effect of synchronizing music playback with the running pace.

\subsection{Questionnaire}

We asked the ten participants to answer a questionnaire consisiting of the following questions:

Q1 Did the music playback synchronized to your pace?

Q2 Did you consciously control the jogging pace according to the music?

Q3 Did you feel that jogging becames easy with our system?

Q4 Do you want to use this system in your daily life?

The questionnaire was conducted on a scale of one to seven.

The results are shown in Fig. 2. The result for Q1 was not high. Our system updates the playback speed at every second based on the avrage pace during last four seconds, so a little latency occured in the synchronization. This would be why the result of Q1 was not high. The result for Q2 was high. This means that synchronization of music playback speed with the jogging pace successfully raised the runner's consciousness of pace control. The results for Q3 and Q4 were also high on average. This means that our system is useful to support jogging by people without frequent exercise.

\section{Conclusion}

We proposed a method for presenting the variation of a jogging pace by synchronizing the music playback speed with the running pace and conducted experiments to confirm the effects of the method. The experimental results showed that this method effectively created steady pace jogging.

In the current implementation, audio signals with various playback speed are necessary to prepare in advance on a PC. Improving this will be one of the most important future issues. To do this, we plan to implement the Phase Vocoder [7] and achieve the music playback synchronization by generating the audio signal with the target speed in real time. In addition, we plan to distribute the software via Google Play and collect a lot of users' feedback for further improvement. 


\section{References}

1. Makikawa, M., et al.: Portable Jogging Monitor Device and its Application for Health Management. In: Proc. APBME 2003 (2003)

2. Glaros, C., et al.: A Wearable Intelligent System for Monitoring Health Condition and Rehabilitation of Running Athletes. In: Proc. IEEE-ITAB 2003 (2003)

3. Mueller, F.F., et al.: Jogging over a Distance. In: Proc. CHI 2007 (2007)

4. Buttussi, F., et al.: MOPET: A Context-aware and User-adaptive Wearable System for Fitness Training. Artificial Intell. Med. 42, 153-163 (2008)

5. Rubisch, J., et al.: A Mobile Music Concept as Support for Achieving Target Heart Rate in Preventive and Recreational Endurance. In: Proc. Audio Mostly Conf. (2010)

6. Sakata, N., et al.: Situated Music: An Application to Interactive Jogging. In: Proc. ISWC 2006 (2006)

7. Flanagan, J.L., et al.: Phase Vocoder. Bell System Technical Journal 45, 1492-1509 (1966)

8. MARY Text To Speech, http://mary.dfki.de/

9. Goto, M., et al.: RWC Music Database: Music Genre Database and Musical Instrument Sound Database. In: Proc. ISMIR 2003 (2003) 